\title{
In Vitro Effect of Gold or Silver Nanoparticles on Meiotic and Postmeiotic Fractions of Rat Germinal Cells
}

\author{
G. Collodel ${ }^{1, *}$, G. Terzuoli ${ }^{1}$, L. Mazzi ${ }^{1}$, N. A. Pascarelli ${ }^{2}$, T. Renieri ${ }^{1}$ and E. Moretti ${ }^{1}$ \\ ${ }^{1}$ Department of Molecular and Developmental Medicine, ${ }^{2}$ Departement of Neurological Sciences Medical and Surgical, \\ University of Siena, Ospedale Santa Maria alle Scotte, viale Bracci, 53100 Siena, Italy
}

\begin{abstract}
The cytotoxicity of Au or Ag nanoparticles (NPs) on rat germinal cells was investigated in vitro. Rat germ cells separated by the STAPUT method in two different populations, pachytene spermatocytes (F5) and round spermatids (F3), were incubated $\left(37^{\circ} \mathrm{C}, 60^{\prime}-120^{\prime}\right)$ with $60 \mu \mathrm{M}, 125 \mu \mathrm{M}, 250 \mu \mathrm{M}$ and $500 \mu \mathrm{M}$ of Au/Ag-NPs. Cell viability was assessed with the Eosin Y test. Au or Ag-NPs were investigated with FEG-STEM/EDS and TEM. A dose-dependent effect on the viability of F3 and F5 populations was observed after incubation with Au-NPs or Ag-NPs $(\mathrm{P}<0.001)$. A significant decrease in cell viability was observed in $\mathrm{F} 5$ fractions compared to $\mathrm{F} 3$ fractions $(\mathrm{P}<0.05)$, except for the samples treated with $60 \mu \mathrm{M}$ of Au-NPs, and the decrease was also significant in all the samples treated with Ag-NPs compared to those incubated with Au-NPs $(\mathrm{P}<0.05)$. Au-NPs were localized in spermatocytes and spermatids whereas Ag-NPs were undetectable. In conclusion, Au- NPs and Ag-NPs seem to exert a negative effect on rat germ cells, particularly on spermatocytes, that appeared significantly more compromised than spermatids. Further research is needed, mainly to carefully explore the possible genotoxicity of these NPs on germinal cells.
\end{abstract}

Keywords: Au or Ag NPs, rat germ cells, cell viability, NPs localisation.

\section{INTRODUCTION}

Nanoparticles (NPs) are substances ranging from 1 to $100 \mathrm{~nm}$ in size. The rapid development of nanotechnologies has given rise to broad applications of NPs in biomedicine. Nanomaterials are already being used in electronics, the food industry, and in cosmetics, and NPs are promising agents for fluorescence and magnetic resonance imaging, drug delivery systems and other applications in medicine. The important antimicrobial activities of silver (Ag) NPs have mainly led to their increasing use in several industrial sectors [1].

Gold ( $\mathrm{Au}$ ) NPs can penetrate cells by endocytosis, and they can be carriers of pharmaceuticals, nucleic acids, and other biologically active substances [2].

Despite the wide application of nanomaterials, there has been a serious lack of information concerning the impact of NPs on human health and the environment. It is believed that the chemical nature, particle size, morphology, and surface chemistry of NPs are key parameters that influence their toxicity, Thus the field of nanotoxicology still lacks the necessary information and clarifications for achieving true risk assessment. In order to optimise the beneficial effects of NP applications, it is essential to understand the fundamental interactions of NPs with biological systems [3].

It is known that NPs are able to penetrate into reproductive tissue through biological barriers; therefore, they may damage various cells: for example, they could reduce sperm

*Address correspondence to this author at the Department of Molecular and Developmental Medicine, University of Siena, Policlinico Santa Maria alle Scotte, Viale Bracci, 53100 Siena, Italy; Fax: 39-0577-233527;

Email: E-mail: giulia.collodel@unisi.it viability and alter cell functions, as well as embryo development [4]. Although the potential toxic effect of NPs on the reproductive sphere is conceivable, further insights are needed in order to clarify this issue [5].

The results of in vivo studies have shown adverse effects of carbon NPs on the male reproductive systems of adult mice [6] and after exposure during foetal life [7]. In addition, the prenatal exposure of rats to diesel exhaust led to endocrine system disruption after birth and suppression of testicular function [8]. Further recent confirmation of the potential toxic effects of NPs on spermatogenesis was reported by Gromadzka-Ostrowska et al. [9] in a study of intravenous administration of Ag-NPs in male rats, which observed a size, dose- and time-dependent decrease of the epididymal sperm count, increased levels of DNA damage in germ cells and a change in testis seminiferous tubule morphometry. Although in vivo testing will continue to provide the most relevant information on human hazards, rapid and validated alternative models of in vitro cytotoxicity are attractive in order to reduce the burden of animal testing [10].

Specifically in the male reproductive field, several in $v i$ tro studies have been performed in different cell models, using various NPs. A dose-dependent toxicity caused by different NPs was observed in mouse spermatogonia [11]; for instance, titanium dioxide and carbon black NPs affected the viability, proliferation and gene expression of mouse Leydig cells [12].

Makhluf et al. [13] demonstrated that magnetite NPs, loading spontaneously into bovine sperm cells, did not affect their functionality. Recently, the in vitro toxicity of Au NPs was even demonstrated in mouse epididymal sperm [14]. In humans, studies have been even rarer. Au-NPs have been 
found to affect human sperm motility and to penetrate into the head and tail of spermatozoa [15]. A recent study by our group [16] demonstrated a significant dose-dependent effect of Au-NPs and Ag-NPs on human sperm motility and viability, although the real damage to sperm occurred using high NPs concentrations $(250-500 \mu \mathrm{M})$ that are probably difficult to reach in vivo.

This study was planned to better investigate the effect that NPs can exert on spermatogenesis; thus, we used $\mathrm{Au}-$ NPs or Ag-NPs in another cell model, rat germ cells separated by the STAPUT method in two different populations, pachytene spermatocytes and round spermatids. Cell viability was assessed with the Eosin Y test and cell morphology was determined by transmission electron microscopy (TEM). NPs were characterized and localized with field emission gun-based scanning transmission electron microscope/energy dispersive spectroscopy and TEM.

\section{MATERIAL AND METHODS}

\section{Characterisation of NPs}

NPs were kindly provided by Cericol Colorobbia Research Center, Sovigliana Vinci, Firenze (Italy). The NPs suspensions were prepared by polyol-mediated synthesis using a bottom-up approach and an organometallic precursor of silver. By employing this procedure, metal NPs can be dispersed in water by means of a nucleation of metallic salts. The process involves an initial phase in which reagents are added in a high-temperature environment of a reaction containing a solvent, such as glycolic solvent medium, with a high capacity for complexing. The next step is the crystal growth phase, performed by mixing the reagents, including surfactants such as polyvinylpyrrolidone (PVP), at room temperature. The obtained NPs are stable for long periods of time without showing aggregation or changes in chemical characteristics. The concentration of the stock solutions of NPs was $1 \%$ (corresponding to $0.0510 \mathrm{M}$ for Au-NPs and $0.0930 \mathrm{M}$ for Ag-NPs) in distilled $\mathrm{H}_{2} \mathrm{O}$ and PVP $(<1 \%)$ as stabilising agent. The concentrations of the stock NPs suspensions was determined using the analysis inductively coupled plasma optical emission spectrometry (ICP-OES). To determine NPs morphology, after a 10 min sonication, a drop of NPs suspension was placed on a formvar-carbon coated grid. After $45 \mathrm{~s}$ the grid was dried with blotting paper and examined at the electron microscope. The hydrodynamic dimensions were determined by Dynamic Light Scattering (Nano-S model by Malvern) and the average size of Au-NPs resulted 50nm and Ag-NPs of $65 \mathrm{~nm}$. Z-potential was -20 $\mathrm{mV}$. For each NPs a blank solution was tested, composed from the stock solution (distilled $\mathrm{H}_{2} \mathrm{O}$ and PVP $<1 \%$ ) and the culture medium without NPs. Using Eosin Y test no cytotoxicity was observed. A $10 \%$ DMSO solution was used as positive control.

\section{Rat Germ Cell Isolation}

Rat germ cells were obtained from three 35-day-old Wistar rats (100 g of body weight) (Charles River, Calco, Italy). Rats were sacrificed by cervical dislocation and testes were collected and placed in Minimum Essential Medium (MEM) supplemented with pyruvate and lactate. After removal of the tunica albuginea, testes were placed in culture medium containing collagenase (activity 0.450 unity $/ \mathrm{ml}$ ) and incubated for $10 \mathrm{~min}$ to remove the interstitium, which was discarded. A second incubation in a shaking water bath at $32^{\circ} \mathrm{C}$ for $45 \mathrm{~min}$ with the same enzymes and $0.1 \%$ Bovine Serum Albumin and DNAse was performed to partially digest the basal lamina of tubules [17]. The cell suspension obtained after enzymatic digestion was used for NPs treatment. The experiment was repeated three times. The study was approved by the Ethics Committee of the University of Siena (CEL AOUS 21-10-2009).

\section{STAPUT Method}

The cell suspension obtained after enzymatic digestion, as described above, was centrifuged and fractionated by velocity sedimentation at unit gravity on a $0.5 \%-3 \%$ albumin gradient (STAPUT method). The two collected and purified cellular fractions were made up of pachytene spermatocytes (F5) and early spermatids (F3), which were then incubated with $\mathrm{Au}$ or Ag NPs. The experiment was repeated three times.

\section{Experimental Design}

$\mathrm{F} 5$ and $\mathrm{F} 3$ were incubated at $37^{\circ} \mathrm{C}$ for 60 and $120 \mathrm{~min}$ in MEM with $60,125,250$ and $500 \mu \mathrm{M}$ of $\mathrm{Au}$ or Ag NPs; cell viability was evaluated by eosin $\mathrm{Y}$ dye exclusion test. Controls (CTR) were samples treated with the same procedure, but without NPs.

\section{Transmission Electron Microscopy (TEM) and FEG- STEM/EDS}

Rat spermatocytes and spermatids incubated with 250 $\mu \mathrm{M}$ Au-NPs or Ag-NPs were fixed in cold Karnovsky fixative and maintained at $4{ }^{\circ} \mathrm{C}$ for $2 \mathrm{~h}$. Fixed specimens were washed in $0.1 \mathrm{~mol} / 1$ cacodylate buffer $(\mathrm{pH} \mathrm{7.2)}$ for $12 \mathrm{~h}$, postfixed in $1 \%$ buffered osmium tetroxide for $1 \mathrm{~h}$ at $4{ }^{\circ} \mathrm{C}$, then dehydrated and embedded in Epon Araldite. Ultra-thin sections were cut with a Supernova ultramicrotome (Reickert Jung, Vienna, Austria), mounted on copper grids for TEM analyses and on nickel grids for Field Emission Gun-based Scanning Transmission Electron Microscope/Energy Dispersive Spectroscopy (FEG-STEM/EDS, model Supra40 by Zeiss Oberkoken, Germany) available at Colorobbia Industry (Montelupo Fiorentino, Florence).

Specimens for TEM were stained with uranyl acetate and lead citrate. Samples were observed and photographed with a Philips CM12 EM 208 transmission electron microscope (TEM; Philips Scientifics, Eindhoven, The Netherlands).

The samples on nickel grids were observed with FEGSTEM/EDS, that shows the material microstructures at atomic resolution, determines crystal structure (atomic arrangements) and analyses chemical composition with a spatial resolution on the order of nanometers. A semiquantitative analysis of NPs concentrations in the examined samples can be derived from a specific spectrum after FEGSTEM/EDS application.

\section{Statistical Analysis}

Comparisons between the percentages of rat germ cells (two fractions F3 and F5) viability measured at 60 and 120 
min, at each concentration of Au-NPs or Ag-NPs, were performed with the Wilcoxon matched pairs test.

The effects of both NPs concentrations on viability were evaluated using the Spearman correlation coefficient (r).

The Wilcoxon matched pairs test was then used to compare:

a the percentages of viability measured in samples treated with $\mathrm{Au}-\mathrm{NPs}$ with those measured in samples treated with Ag-NPs in both F3 and F5 fractions, for each NPs concentration;

b the percentages of viability of F3 treated with Au-NPs with those measured in F5 treated with Au-NPs, and the percentages of viability of F3 treated with Ag-NPs with those measured in F5 treated with Ag-NPs, at each NPs concentration used;

c the control with each treated sample.

An A P value of $<0.05$ (two-tailed) was considered statistically significant. All analyses were performed using SPSS Statistical Software_Version 20 (SPSS Inc., Chicago, USA).

\section{RESULTS}

\section{Evaluation of Rat Germ Cell Viability After NPs Incuba- tion}

We tested the possible cytotoxicity of Au-NPs or Ag-NPs on rat germ cells fractioned as a meiotic population composed of pachytene spermatocytes (F5) and a post meiotic fraction of early spermatids (F3). The effects of Au-NPs or Ag-NPs on cell viability were determined with the eosin Y test. We did not find significant differences between the cell viability percentages in the germ cell populations incubated with either of the NPs at 60 or $120 \mathrm{~min}(\mathrm{P}>0.05)$; for this reason, we reported the results obtained after $60 \mathrm{~min}$ of incubation.
A dose-dependent effect on the viability of F3 and F5 populations was observed after incubation with Au-NPs (F3: $\mathrm{r}=-0.9625, \mathrm{P}<0.001 ; \mathrm{F} 5: \mathrm{r}=-0.9716, \mathrm{P}<0.001)$ or Ag-NPs (F3: $r=-0.972, \mathrm{P}<0.001 ; \mathrm{F} 5: \mathrm{r}=-0.971, \mathrm{P}<0.001$ ) as shown in (Fig. 1). In both fractions, F5 and F3, the percentages of cell viability measured in all samples treated with Au-NPs were significantly higher than those observed in specimens incubated with Ag-NPs $(\mathrm{P}<0.05$; Fig. 1). In addition, we compared the percentages of cell viability between F5 and F3 separately for each sample treated with Au or Ag. A significant decrease in cell viability was observed in F5 fractions compared to $\mathrm{F} 3$ fractions $(\mathrm{P}<0.05)$, except for the samples treated with $60 \mu \mathrm{M} \mathrm{Au}-\mathrm{NPs}$ (Fig. 1).

A significant decrease in cell viability was detected in all samples treated with each concentration of $\mathrm{Au}$ or Ag-NPs compared to controls $(\mathrm{CTR})(\mathrm{P}<0.05)$, except for specimens incubated with Au-NPs $60 \mu \mathrm{M}$ (Fig. 1).

\section{Localisation of NPs on Rat Germ Cells by TEM and X Ray Microanalysis}

After evaluating the cytotoxicity of $\mathrm{Au}$ or Ag-NPs, we investigated their presence in germ cells. F5 and F3 germ cell fractions were composed of cells with a generally normal morphology.

In the F5 fractions treated with Au-NPs, rare spermatocytes showed NPs aggregates in the plasma membrane and cytoplasm (Fig. 2); in the F3 fractions, rare early spermatids showed the same type of aggregates in the plasma membrane (Fig. 3).

A further demonstration that Au-NPs are able to interact with cells was shown in the use of FEG-STEM/EDS. Using this method, a signal of an Au element was highlighted in some areas of cytoplasm in cells treated with Au-NPs (Fig. 4a-4a'). We did not observe the aggregates in any of the examined control samples. Ag-NPs were undetectable in both fractions, by TEM or by FEG-STEM/EDS.

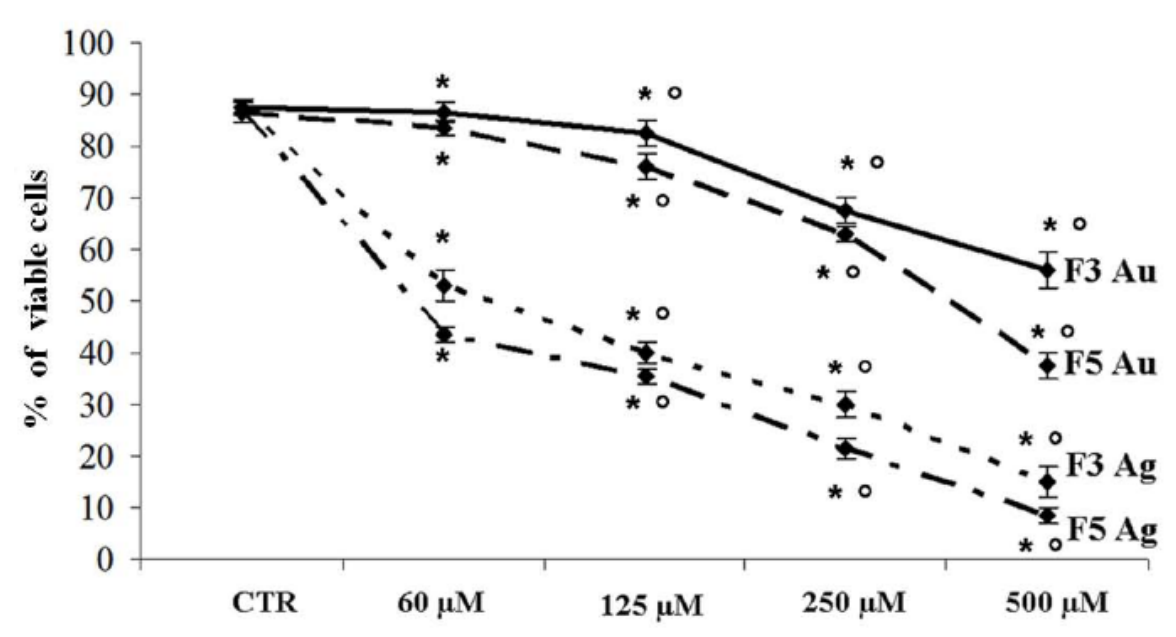

\section{Concentrations}

Fig. (1). Mean and SD of the percentages of viable rat spermatocytes (F5) and spermatids (F3) treated with Au or Ag NPs at different concentrations. CTR controls, same conditions without NPs. ${ }^{\circ} \mathrm{P}<0.05$ : each sample F3 vs F5 at the same concentration with both NPs; * P $<0.05$ : each sample (F3 or F5) treated with Au-NPs versus the respective treated with Ag-NPs at the same concentration. 


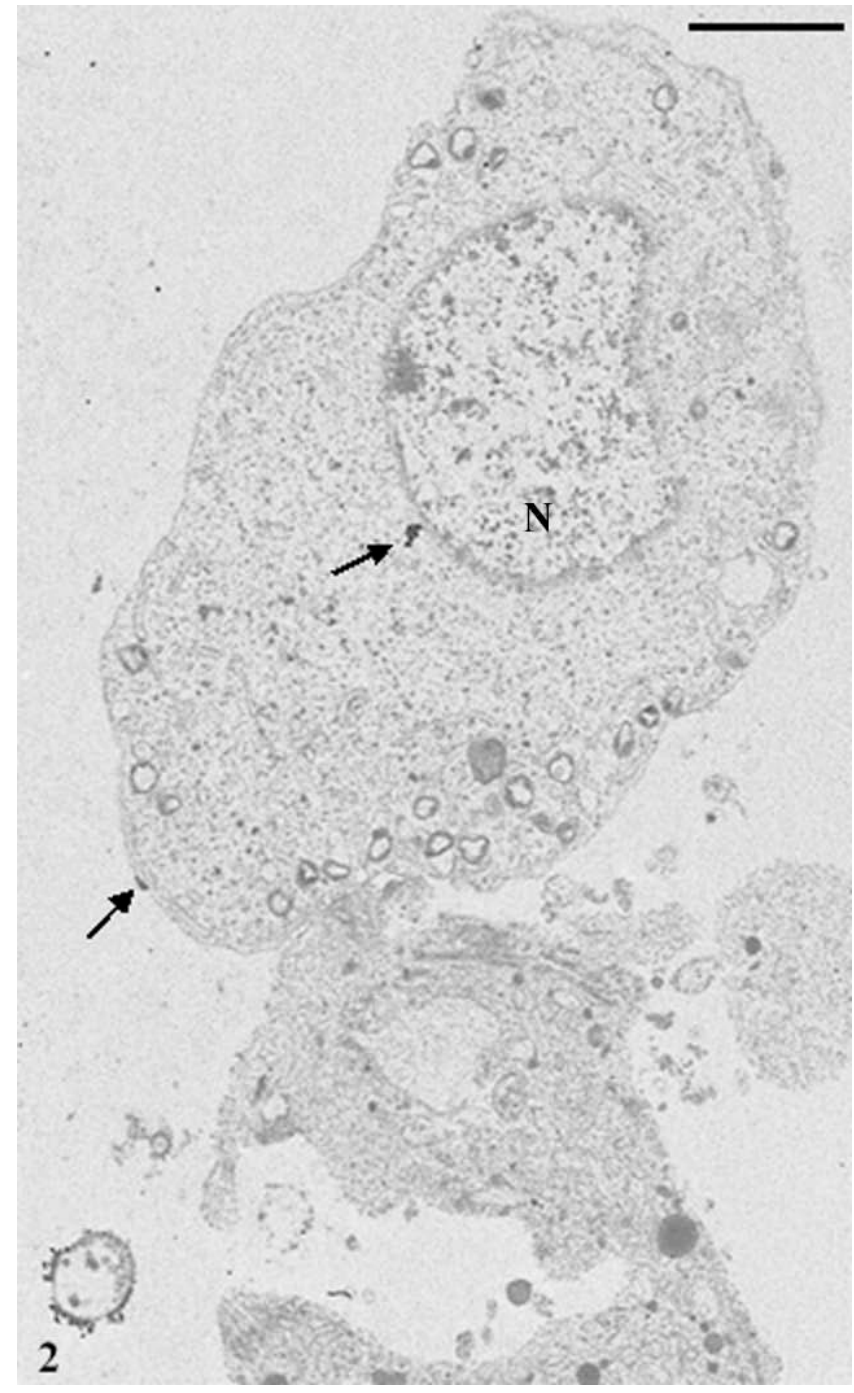

Fig. (2). TEM micrograph of a section of rat spermatocyte. Au-NPs aggregates were present in the plasma membrane and cytoplasm (arrows). Nucleus (N). Bar $=1 \mu \mathrm{m}$.

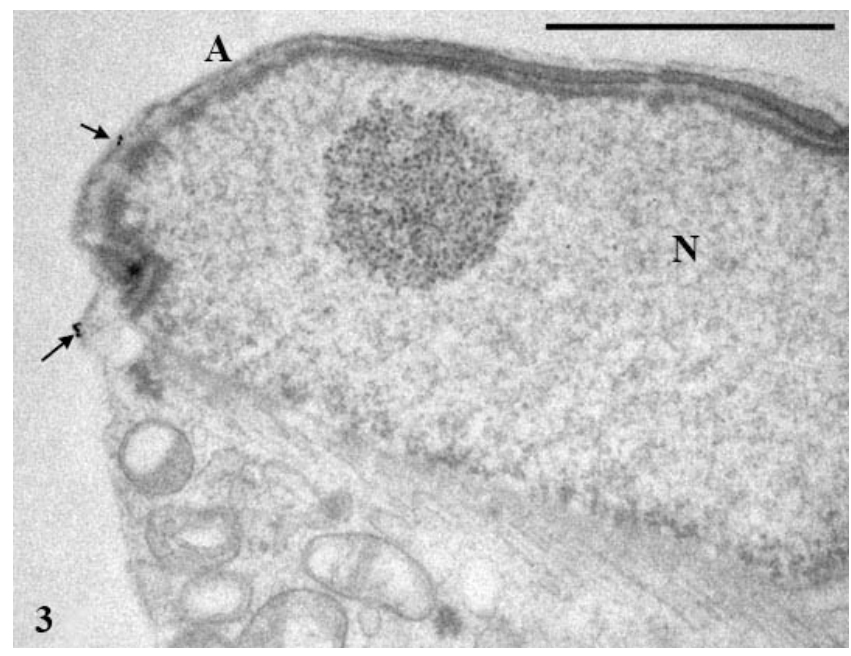

Fig. (3). TEM micrograph of a section of rat spermatid incubated with Au-NPs, some aggregates of particles were detected in the plasma membrane (arrows). Acrosome (A), Nucleus $(\mathrm{N})$. Bar $=1$ $\mu \mathrm{m}$.


Fig. (4). Identification of Au-NPs using FEG-STEM/EDS: EDS spectrum (a) signal of an $\mathrm{Au}$ element was highlighted in cells treated with Au-NPs (a').

\section{DISCUSSION}

This research was designed to explore the in vitro effects of $\mathrm{Au}$ or Ag-NPs in two fractions of rat germinal cells; spermatids and spermatocytes. The choice of NPs was based on the fact that $\mathrm{Au}$ and $\mathrm{Ag}$ can be easily identified if they are incorporated into the cells, since they are not physiologically present in germ cells. To choose the concentrations used in these experiments, we considered the results obtained by our group in a recent study of the effects of such NPs on ejaculated human sperm [16]; in the present study we were interested in exploring the toxic effects of $\mathrm{Au}$ or Ag NPs on testicular germ cells, in particular, their possible ability to penetrate inside the cells, and in defining their localisation. We chose rat germ cells mainly due to the difficulty of working with human germ cells and to take advantage of the existing method for purifying the meiotic and post meiotic fractions from rat testis [17], excluding all other cells.

The significant dose dependent cytotoxic effect of both NPs on both fractions of germ cells was demonstrated and the Ag-NPs appeared to be significantly more harmful for meiotic and post meiotic sperm fractions than Au-NPs. The detrimental role of Ag-NPs, coated with hydrocarbon and polysaccharide, in mouse spermatogonia was reported by Braydich-Stolle et al. [11] who demonstrated in vitro an increase in apoptosis and ROS production after Ag-NPs $(>10$ $\mu \mathrm{g} / \mathrm{ml}$ ) treatment for 24-48 $\mathrm{h}$. In addition, the cytotoxic and apoptotic effects of Ag-NPs had also been demonstrated in HeLa human cells [18]. Recently, the toxicity and the in- 
flammogenic potential of Ag-NPs were confirmed in vivo in rats, mice and plants and in vitro in several cell lines [19, 20]. In the human testicular embryonic carcinoma cell line and in mouse primary testicular cells, Ag-NPs $(20 \mathrm{~nm}$ and $200 \mathrm{~nm}$ ) caused apoptosis, necrosis and decreased proliferation in a concentration- and time-dependent manner [21]. The vast majority of trials regarding Au-NPs cytotoxicity have been performed using somatic cell culture lines. The results have shown a considerable dependency of toxic effects on size and NPs surface modifications; however, a recent study of gametes showed a dose-dependent sensitivity of spermatozoa towards Au-NPs [4].

In the present study, a comparison between percentages of cell viability in meiotic and post meiotic fractions, for each concentration of Au-NPs or Ag-NPs used, was performed in order to highlight a possible difference in sensitivity between these two cell populations. Thus, in rat germ cells the meiotic fraction, the pachytene spermatocyte, was more vulnerable to treatment with both NPs compared to the early spermatid fraction. Spermatocytes are cells in active meiosis; therefore, it is plausible that they are more susceptible to the cytotoxic effects of NPs compared to spermatids, which are post-meiotic cells. This different susceptibility of the two fractions of rat germ cells was observed by our group in other experiments, in which an in vitro induced oxidative stress was more evident in spermatocytes than in spermatids [22]. The other main goal of this research concerned the localizations of NPs and their visualization directly in the cells. Au-NPs were localized by both TEM and FEG-STEM/EDS outside and inside both spermatocytes and spermatids. Analysing the ultrastructure, no particular cell morphological damage seems to occur in the cells of both fractions. The behaviour of Ag-NPs, that were undetectable in all observed samples by FEG-STEM/EDS or TEM, was different, as we had already observed in human spermatozoa [16] and in osteoarthritis chondrocytes [23]. In that case, we hypothesised that the high concentration of Ag-NPs could increase the release of $\mathrm{Ag}+$ ions [24] that may easily penetrate into the cells and interfere with the cell structures. Recently Kruszewski et al. [25], in 3 human cell lines treated with $20 \mathrm{~nm}$ or $200 \mathrm{~nm} \mathrm{Ag-NPs} \mathrm{observed} \mathrm{no} \mathrm{silver} \mathrm{ions} \mathrm{in} \mathrm{the}$ medium after $2 \mathrm{~h}$ incubation and only $0.018 \mu \mathrm{g} / \mathrm{mL}$ silver ions were released from $100 \mu \mathrm{g} / \mathrm{mL}$ Ag-NP solution after $24 \mathrm{~h}$. These data may suggest only a neglectable role of silver ions in oxidative DNA damage. It also cannot be excluded that Ag-NPs could exert their negative effects without being internalized in the cells, and that they could be easily removed after the numerous steps required for TEM preparation. Park et al. [26] suggested that the potency of silver in the form of nanoparticles to induce cell damage compared to silver ions is cell type- and size-dependent.

Other studies have reported transmission electron microscopy images showing that Au-NPs and Ag-NPs are both trapped in vesicles in the cytoplasm of macrophages [27] and that Ag-NPs are efficiently internalized via scavenger receptor-mediated phagocytosis in murine macrophages [28]. In addition, NPs could interfere with membrane receptors or with cell signalling involved in viability maintenance. Braydich-Stolle et al. [11] demonstrated that Ag-NPs are able to induce a significant decline in spermatogonia stem cell proliferation, disrupting GDNF/Fyn kinase signalling. Comfort et al. [29] showed an interference of $\mathrm{Ag}, \mathrm{Au}$ and iron oxide NPs on epidermal growth factor signal transduction in epithelial cells.

Recent in vitro and in vivo studies [20] indicate that AgNPs show genotoxic effects, also in mammalian cell system [30]. Moreover Balansky et al. [31] demonstrated the transplacental size-dependent clastogenic and epigenetic effects of AuNPs in mouse fetus.

Although we are aware that these are only preliminary studies, we suggest that engineered Au-NPs and Ag-NPs seem to exert a negative effect on rat germ cells, particularly on the spermatocytes that appeared significantly more compromised than spermatids. These data became relevant also after than other authors reported that different NPs can penetrate the testicular barrier [32-34].

Further in vitro and in vivo research, focussed on the possible toxicity and genotoxicity of these NPs on germinal cells, is needed.

\section{CONFLICT OF INTEREST}

The authors confirm that this article content has no conflicts of interest.

\section{ACKNOWLEDGEMENT}

The authors would like to thank Dr. Giovanni Baldi, Dr. Arianna Signorini, Dr. Marco Trassinelli (Colorobbia, Empoli), Dr. Cristiana Giordano, and Dr. Cristina Salvatici (CE.M.E, CNR, Sesto Fiorentino, Florence) for their technical and scientific contributions to the study.

\section{REFERENCES}

[1] de Lima R, Seabra AB, Durán N. Silver nanoparticles: a brief review of cytotoxicity and genotoxicity of chemically and biogenically synthesized nanoparticles. J Appl Toxicol 2012; 32: 867-79.

[2] Zielinska M, Sawosz E, Grodzik M, et al. Effect of heparan sulfate and gold nanoparticles on muscle development during embryogenesis. Int J Nanomed 2011; 6: 3163-72.

[3] Johnston HJ, Hutchison G, Christensen FM, Peters S, Hankin S, Stone $\mathrm{V}$. A review of the in vivo and in vitro toxicity of silver and gold particulates: particle attributes and biological mechanisms responsible for the observed toxicity. Crit Rev Toxicol 2010; 40: 328-46.

[4] Taylor U, Barchanski A, Garrels W, et al. Toxicity of Gold Nanoparticles on Somatic and Reproductive Cells. In: Zahavy et al. Eds. Nano-Biotechnology for Biomedical and Diagnostic Research. Advances in Experimental Medicine and Biology. Springer Science+Business Media B.V.733, 2012.

[5] Ema M, Kobayashi N, Naya M, Hanai S, Nakanishi J. Reproductive and developmental toxicity studies of manufactured nanomaterials. Reprod Toxicol 2010; 30: 343-52.

[6] Yoshida S, Hiyoshi K, Ichinose T, et al. Effect of nanoparticles on the male reproductive system of mice. Int J Androl 2009; 32: 337 42.

[7] Yoshida S, Hiyoshi K, Oshio S, Takano H, Takeda K, Ichinose T. Effects of fetal exposure to carbon nanoparticles on reproductive function in male offspring. Fertil Steril 2010; 93:1695-9.

[8] Li C, Taneda S, Taya K, et al. Effects of in utero exposure to nanoparticle-rich diesel exhaust on testicular function in immature male rats. Toxicol Lett 2009; 185: 1-8.

[9] Gromadzka-Ostrowska J, Dziendzikowska K, Lankoff A, et al. Silver nanoparticles effects on epididymal sperm in rats. Toxic Lett 2012; 214: 251-8.

[10] Maynard AD, Aitken RJ, Butz T, et al. Safe handling of nanotechnology. Nature 2006; 444: 267-9. 
[11] Braydich-Stolle L, Hussain S, Schlager JJ, Hofmann MC. In vitro cytotoxicity of nanoparticles in mammalian germline stem cells. Toxicol Sci 2010; 88: 412-9.

[12] Komatsu T, Tabata M, Kubo-Irie M, et al. The effects of nanoparticles on mouse testis Leydig cells in vitro. Toxicol in vitro 2008; 22: $1825-31$

[13] Makhluf SB, Qasem R, Rubinstein S, Gedanken A, Breitbart H. Loading magnetic nanoparticles into sperm cells does not affect their functionality. Langmuir 2006; 22: 9480-2.

[14] Zakhidov ST, Marshak TL, Malolina EA, et al. Gold nanoparticles disturb nuclear chromatin decondensation in mouse sperm in vitro. Biochemistry (Moscow) Supplement Series A: Membrane and Cell Biology 2010; 4: 293-6.

[15] Wiwanitkit V, Sereemaspun A, Rojanathanes R. Effect of gold nanoparticles on spermatozoa: the first world report. Fertil Steril 2009; 91: e7-8.

[16] Moretti E, Terzuoli G, Renieri T, et al. In vitro effect of gold and silver nanoparticles on human spermatozoa. Andrologia 2012; doi:10.1111/and.12028

[17] Chiarella P, Puglisi R, Sorrentino V, Boitani C, Stefanini M. Ryanodine receptors are expressed and functionally active in mouse spermatogenic cells and their inhibition interferes with spermatogonial differentiation. J Cell Sci 2004; 15: 4127-34.

[18] Miura N, Shinohara Y. Cytotoxic effect and apoptosis induction by silver nanoparticles in HeLa cells. Biochem Biophys Res Commun 2009; 390: 733-7.

[19] Gaiser BK, Hirn S, Kermanizadeh A, et al. Effects of Silver Nanoparticles on the Liver and Hepatocytes in vitro. Toxicol Sci 2013; 131(2): 537-47.

[20] Ghosh MJM, Sinha S, Chakraborty A, Mallick SK, Bandyopadhyay M, Mukherjee A. In vitro and in vivo genotoxicity of silver nanoparticles. Mutat Res 2012; 749: 60-9.

[21] Asare N, Instanes C, Sandberg WJ, et al. Cytotoxic and genotoxic effects of silver nanoparticles in testicular cells. Toxicology 2012; 291: 65-72.

[22] Collodel G, Federico MG, Geminiani M, et al. Effect of transresveratrol on induced oxidative stress in human sperm and in rat germinal cells. Reprod Toxicol 2011;31: 239-46.
[23] Pascarelli NA, Moretti E, Terzuoli G, et al. Effects of gold and silver nanoparticles in cultured human osteoarthritic chondrocytes. J Appl Toxicol, 2013; 33(12): 1506-13.

[24] Xiu ZM, Ma J, Alvarez PJ. Differential Effect of Common Ligands and Molecular Oxygen on Antimicrobial Activity of Silver Nanoparticles versus Silver Ions. Environ Sci Technol 2011; 45: 9003-8.

[25] Kruszewski M, Grądzka I, Bartłomiejczyk T, et al. Oxidative DNA damage corresponds to the long term survival of human cells treated with silver nanoparticles. Toxicol Lett 2013; 219:151-9.

[26] Park MV, Neigh AM, Vermeulen JP, et al. The effect of particle size on the cytotoxicity, inflammation, developmental toxicity and genotoxicity of silver nanoparticles. Biomaterials 2011; 32: 9810-7.

[27] Yen HJ, Hsu SH, Tsai CL. Cytotoxicity and immunological response of gold and silver nanoparticles of different sizes. Small 2009; 5: 1553-61.

[28] Singh RP, Ramarao P. Cellular uptake, intracellular trafficking and cytotoxicity of silver nanoparticles. Toxicol Lett 2012;213: 24959.

[29] Comfort KK, Maurer EI, Braydich-Stolle LK, Hussain SM. Interference of silver, gold, and iron oxide nanoparticles on epidermal growth factor signal transduction in epithelial cells. ACS Nano 2011;27: 10000-8

[30] Kim HR, Park YJ, Shin da Y, Oh SM, Chung KH. Appropriate in vitro methods for genotoxicity testing of silver nanoparticles. Environ Health Toxicol 2013; 28: e2013003.

[31] Balansky R, Longobardi M, Ganchev G, et al. Transplacental clastogenic and epigenetic effects of gold nanoparticles in mice. Mutat Res 2013 Sep 1. pii: S0027-5107(13)00128-0.

[32] Kim JS, Yoon TJ, Yu KN, et al. Toxicity and tissue distribution of magnetic nanoparticles in mice. Toxicol Sci 2006; 89: 338-47.

[33] Morishita Y, Yoshioka Y, Satoh $\mathrm{H}$, et al. Distribution and histologic effects of intravenously administered amorphous nanosilica particles in the testes of mice. Biochem Biophys Res Commun 2012; 420: 297-301

[34] Zhou L, Yang WX. Nanoparticles and spermatogenesis: how do nanoparticles affect spermatogenesis and penetrate the blood-testis barrier. Nanomedicine 2012; 7: 579-96.

(C) Collodel et al.; Licensee Bentham Open.

This is an open access article licensed under the terms of the Creative Commons Attribution Non-Commercial License (http://creativecommons.org/licenses/by-nc/3.0/) which permits unrestricted, non-commercial use, distribution and reproduction in any medium, provided the work is properly cited. 